\title{
A Morphology Study on Thermoelectric Al-Substituted ZnO
}

\author{
Nina Schäuble ${ }^{\mathrm{a}}$, Benjamin E. Süess ${ }^{\mathrm{a}}$, Sascha Populoh ${ }^{\mathrm{a}}$, Anke Weidenkaff ${ }^{\mathrm{a}}$, \\ Myriam H. Aguirre ${ }^{\mathrm{a}}$ \\ ${ }^{a}$ Laboratory of Solid State Chemistry and Catalysis \\ Empa, Swiss Federal Laboratories for Materials Science and Technology \\ Überlandstrasse 129, CH-8600 Dübendorf, Switzerland \\ *anke.weidenkaff@empa.ch
}

\begin{abstract}
Al-substituted $\mathrm{ZnO}$ is a potential high temperature n-type thermoelectric material. The thermoelectric properties, the morphology and the microstructure of $\mathrm{Zn}_{0.98} \mathrm{Al}_{0.02} \mathrm{O}$ prepared by soft chemistry synthesis were studied and compared with samples synthesised by solid state reaction. The soft chemistry method produces samples with better connectivity of the grains and less structural defects leading to a lower electrical resistivity and a higher thermal conductivity than solid state reaction. The Seebeck coefficient is not affected by the microstructure of the material. The highest value of the dimensionless Figure of Merit ZT of 0.21 is therefore reached at $1275 \mathrm{~K}$ for the samples from soft chemistry synthesis.
\end{abstract}

Keywords: Thermoelectric properties, Al-substituted $\mathrm{ZnO}$, thermal cycling.

PACS: 72.20.Pa, 68.37.Og

\section{INTRODUCTION}

Metal oxides have been shown to have promising thermoelectric properties [1]. Beside their good chemical stability in air, many of them consist of abundant elements, are low priced and non-toxic, compared to the state-ofthe-art telluride based thermoelectric materials [1]. This makes them attractive for thermoelectric applications, especially at high temperature. Zinc oxide has been pointed out to be a promising thermoelectric material due to its high Seebeck coefficient. By substituting zinc with $2 \mathrm{~mol} \%$ of Al the electrical resistivity is decreased [2] considering a typical example for n-type doping in semiconductors [3]. The substitution leads to a higher electron concentration and therefore a lower resistivity in the material.

Solid state reaction (SSR) is usually used to synthesise ceramic materials. However, a high sintering temperature is needed for ion diffusion in order to achieve phase formation. In soft chemistry (SC) synthesis the Al and the $\mathrm{Zn}$ ions are mixed in the liquid phase and a network between the ions is formed. The homogeneous distribution of the ions is sustained in the solid phase and the solid solution of $\mathrm{Al}$ in the $\mathrm{ZnO}$ lattice can form without diffusion of the ions. The SC synthesis is suitable for the formation of low-level substituted metal oxides to obtain a homogeneous distribution of the substitution element $[4,5]$.

In this work the thermoelectric properties, the morphology and the microstructure of Al-substituted $\mathrm{ZnO}$ bulk samples are compared for the above mentioned synthesis methods. $\mathrm{Zn}_{0.98} \mathrm{Al}_{0.02} \mathrm{O}$ samples were synthesised by $\mathrm{SC}$ and by SSR in order to measure the thermoelectric properties. A scanning (SEM) and transmission electron microscopy (TEM) study was done to identify morphological and structural discrepancies between samples from both synthesis methods.

\section{EXPERIMENTAL}

Al-substituted $\mathrm{ZnO}$ was prepared by soft chemistry synthesis (SC) and by solid state reaction (SSR). SC $\mathrm{Zn}_{0.98} \mathrm{Al}_{0.02} \mathrm{O}$ was produced by thermal decomposition of the corresponding polymeric citrate precursors [6]. The required amount of $\mathrm{Zn}\left(\mathrm{NO}_{3}\right)_{2} * 6 \mathrm{H}_{2} \mathrm{O}$ (Sigma Aldrich, 98\%) and $\mathrm{Al}\left(\mathrm{NO}_{3}\right)_{3} * 9 \mathrm{H}_{2} \mathrm{O}$ (Sigma Aldrich, puriss. p. a.)

9th European Conference on Thermoelectrics

AIP Conf. Proc. 1449, 421-424 (2012); doi: 10.1063/1.4731586

(C) 2012 American Institute of Physics 978-0-7354-1048-0/\$30.00 
was dissolved in water. Citric acid (Alfa Aesar 99+\%) was added as a chelating ligand to complex the different cations. The solution was homogenised at $373 \mathrm{~K}$ for $4 \mathrm{~h}$ under continuous stirring and reflux. The precursor solution was dried by removing the reflux promoting the formation of a colourless viscous gel. The precursor was heated up decomposed at $573 \mathrm{~K}$ for $2 \mathrm{~h}$. Crystallisation was achieved by calcination of the amorphous xerogel at $1073 \mathrm{~K}$. For the SSR the required amount of $\mathrm{ZnO}$ (Aldrich, nanopowder $<100 \mathrm{~nm}$ ) and $\gamma-\mathrm{Al}_{2} \mathrm{O}_{3}$ was combined $\left(\mathrm{The} \gamma-\mathrm{Al}_{2} \mathrm{O}_{3}\right.$ powder was synthesised by the Department of Molecular and Material Sciences of Interdisciplinary Graduate School of Engineering Science at Kyushu University - Group of Prof. M. Ohtaki). The precursor powders were mixed by planetary milling with $1 \mathrm{~mm} \mathrm{ZrO}_{2}$ balls for $4 \mathrm{~h}$ at $300 \mathrm{rpm}$. The polycrystalline samples from both synthesis methods were pressed into circular pellets and sintered at $1673 \mathrm{~K}$ for $10 \mathrm{~h}$ in air in order to achieve high density.

Phase purity of the samples was investigated by X-Ray diffraction (XRD) with a PANalytical X'pert diffractometer using $\mathrm{Cu}-\mathrm{K} \alpha$ radiation. The morphology and the crystal structure of the calcined powders and the sintered pellets were studied using a SEM Hitachi S-4800 and a TEM Jeol JEM 2000FS TEM/STEM. The cationic composition was investigated by EDS (energy dispersive spectroscopy) in SEM and TEM.

The sintered pellets were used to determine transport and thermal properties. The thermal conductivity $\kappa$ was calculated from the bulk density determined by the geometrical method, the thermal diffusivity measured by the laser flash method using a Netzsch LFA-457 apparatus and the heat capacity measured by differential scanning calorimetry using a Netzsch DSC 404C Pegasus. The transport property measurements were done on bar-shaped pellets cut from the circular pellets with general dimensions of $1.5 \mathrm{~mm} \times 1.5 \mathrm{~mm} \times 8.0 \mathrm{~mm}$. The electrical resistivity and Seebeck coefficient were measured using the RZ2001i measurement system from Ozawa Science, Japan. A treatment of four cycles in air as a function of the temperature from $313 \mathrm{~K}$ to $1275 \mathrm{~K}$ and back to room temperature was done in order to check the stability of the material.

\section{RESULTS AND DISCUSSION}

Fig. 1 shows the electrical resistivity, the Seebeck coefficient and the thermal conductivity (lattice and electronic part) as a function of the temperature measured in air for samples synthesised by soft chemistry (SC) and by solid state reaction (SSR). The values of the Seebeck coefficient are very similar for the two samples from different synthesis methods. However, the resistivity values are twice as high for the samples synthesised by SSR than for the ones by SC at high temperature and four times as high at low temperature. An increase in both transport properties can be observed with cycling, which is comparable for both samples. The first and the last of four cycles are chosen in Fig. 1 in order to illustrate the change in properties with cycling. The increase of the absolute value of the Seebeck coefficient is about $10 \%$. The electrical resistivity increases continuously about $25 \%$ and $20 \%$ over all four cycles for the samples synthesised by SC and SSR, respectively. The evolution of the transport properties can be explained by an uptake of oxygen by the material during the measurement in air atmosphere leading to a decreased electron concentration and an increase in resistivity and absolute Seebeck coefficient values. This change in the transport properties is discussed in [7] in more detail. The thermal conductivity is composed of the sum of a lattice and an electronic part. Fig. 1c shows larger values for the samples synthesised by SC than by SSR for both the lattice and the electronic part. A ZT value of 0.21 is reached at $1275 \mathrm{~K}$ for the samples synthesised by SC, calculated from the first cooling curve for the electrical resistivity and the Seebeck coefficient. The ZT of the SSR prepared samples is 0.13 . The ZT values decrease by $24 \%$ and $31 \%$ respectively during four cycles with a better stability for SC samples compared to SSR samples.

a)

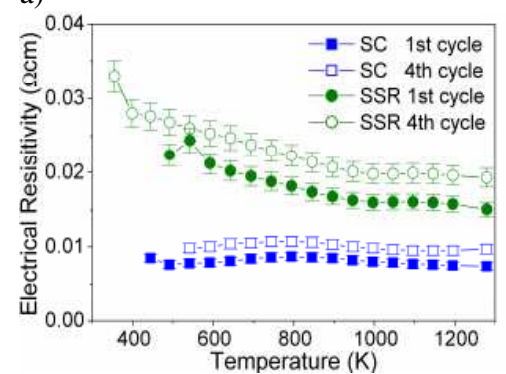

b)

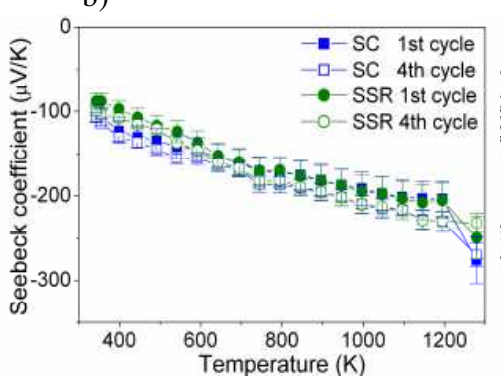

c)

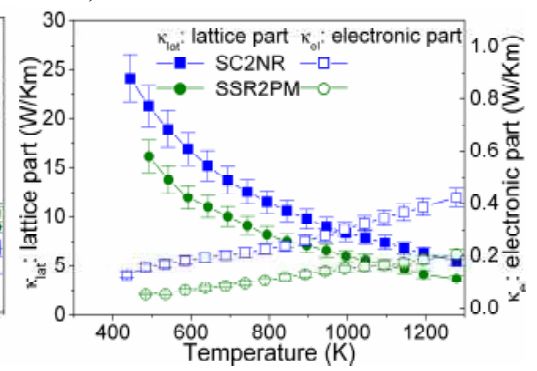

FIGURE 1. a) Electrical resistivity, b) Seebeck coefficient and c) thermal conductivity as a function of the temperature for samples synthesised by SSR and by SC. (First and last of four cooling cycles are shown for resistivity and Seebeck coefficient.) 
The electrical resistivity can either be influenced by the composition and as a consequence by the carrier concentration, or by the morphology of the sample itself. However, the Seebeck coefficient values, which also depend on the carrier concentration, are similar for both samples. Therefore, the difference in electrical resistivity between the samples from the two synthesis methods is due to a variation in morphology rather than its composition. The densities for the two samples are in the same range of about 95\%. SEM and TEM studies were performed in order to analyse the morphology and the microstructure of the two samples.

Fig. 2 shows the morphology in SEM back scattered electron (BSE) micrographs of the samples synthesised by SSR (a) and SC (b) after etching. The grain size is similar with 4 to $50 \mu \mathrm{m}$ for the samples synthesised by SSR and 4 to $60 \mu \mathrm{m}$ for the ones by SC due to the long sintering time. However, SSR leads to more elongated grains, while the samples synthesised by SC show irregular shapes. The dark areas in the micrographs show pores and the dark grey areas consist of a secondary spinel phase of $\mathrm{ZnAl}_{2} \mathrm{O}_{4}$, which as known is likely to be formed in $\mathrm{Al}$-substituted $\mathrm{ZnO}$ [2]. SC leads to a more homogeneous distribution of pores and $\mathrm{ZnAl}_{2} \mathrm{O}_{4}$ particles. In the sample synthesised by SSR there are areas with a smaller and some with a larger amount of pores and $\mathrm{ZnAl}_{2} \mathrm{O}_{4}$ particles (Fig. 2 shows an area with a smaller amount). Overall, the amount of pores and secondary phase particles is slightly larger for SC than for SSR prepared samples. EDX studies show a homogeneous distribution of $\mathrm{Al}$ in the $\mathrm{ZnO}$ matrix for both samples.
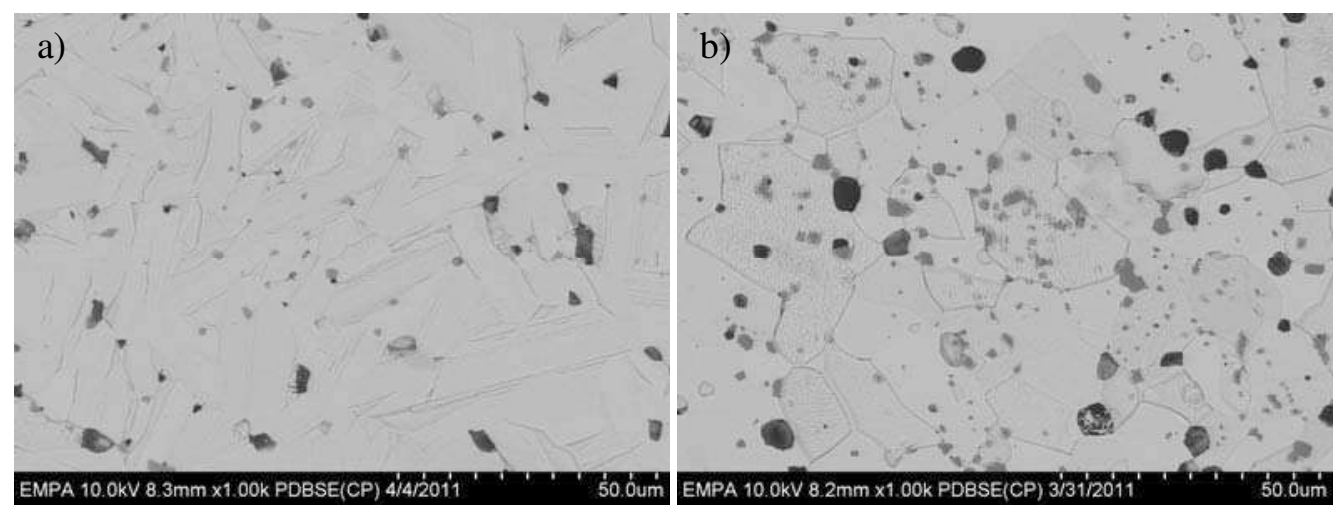

FIGURE 2. SEM BSE images of a) the sample by SSR and b) by SC.

The TEM study reveals a difference in the nanostructure between the samples. Fig. 3 shows TEM micrographs of grains and grain boundaries. Fast Fourier Transform (FFT) images help to show the orientation of the different grains. A better connectivity between the grains and less structural defects in the grains and at grain boundaries are found in the sample synthesised by SC. Some disorder can be observed in the SC sample, especially due to a large change in the orientation between the grains. However, in the sample synthesised by SSR, more defects and also some amorphous phase can be detected at the grain boundaries.

Poorly connected grains and structural defects lead to scattering of the electrons and the phonons in the material. The higher electrical resistivity in the sample synthesised by SSR is induced by this stronger scattering of the electrons at the larger amount of defects. The electronic part of the thermal conductivity can be approximated using the Wiedeman-Franz law $\left(\kappa_{\mathrm{el}}=\mathrm{L} \mathrm{T} / \rho\right.$ (with L the Lorentz number, $\rho$ the electrical resistivity and T the absolute temperature) [2]). The lower electrical part of the thermal conductivity for the sample synthesised by SSR is due to the higher electrical resistivity. The lower values for the lattice part of the thermal conductivity for the samples by SSR are induced by the stronger scattering of the phonons at the defects in the grains and at the grain boundaries.

The length scale of electron and phonon scattering is more in the nanostructural than in the microstructural range. Therefore, the discrepancy in the thermoelectric properties between the samples produced by the different synthesis methods is mainly caused by differences in defects and crystallinity, more than by the microstructural morphology. 


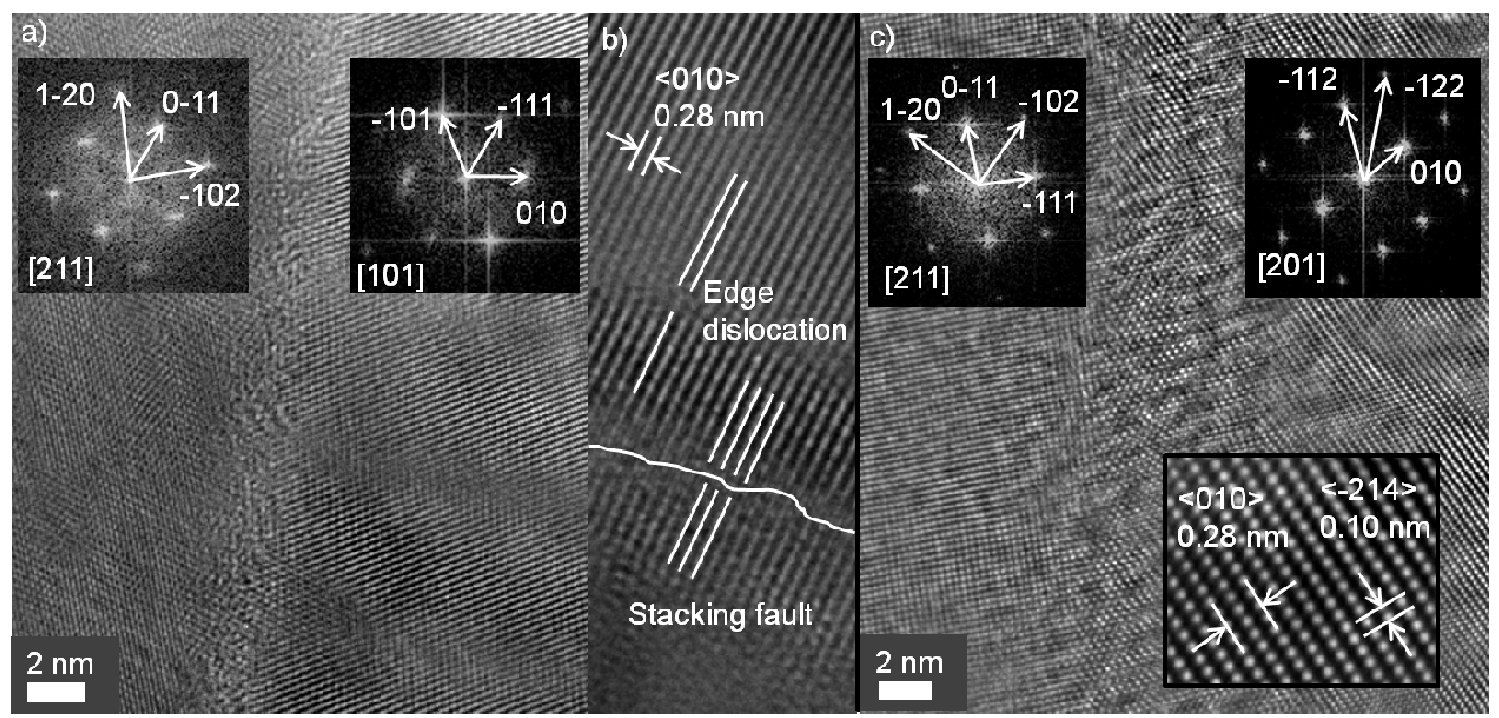

FIGURE 3. TEM micrographs, fast Fourier transform (FFT) images and enlarged insets of a), b) the sample by SSR and c) by SC.

\section{CONCLUSION}

Al-substituted $\mathrm{ZnO}$ was synthesised by soft chemistry (SC) and by solid state reaction (SSR) methods in order to study and compare the thermoelectric properties. SEM and TEM studies reveal a similar morphology but differences in the microstructure. SC produces a better connectivity of the grains and less structural defects at the grain boundaries and within the grains. The defects lead to stronger scattering of the electrons and phonons in the samples prepared by SSR resulting in a higher electrical resistivity and a lower thermal conductivity than for the SC ones. The Seebeck coefficient is not affected by the structural discrepancy. The lower electrical resistivity of the sample synthesised by SC overcompensates the higher thermal conductivity, which would be unfavourable for the thermoelectric performance. Therefore, SC produces material with a higher ZT value of 0.21 compared to 0.13 for the sample synthesised by solid state reaction.

\section{ACKNOWLEDGEMENT}

The authors thank the Swiss National Science Foundation (SNSF) for the financial support (project no. 124688). Many thanks are given to Prof. M. Ohtaki for providing the $\gamma-\mathrm{Al}_{2} \mathrm{O}_{3}$ powder and for many fruitful discussions.

\section{REFERENCES}

1. K. Koumoto et al., "Oxide Thermoelectrics" in Thermoelectrics Handbook, edited by D.M. Rowe, Boca Raton: CRC Press, 2006, p. 35-1.

2. T. Tsubota, M. Ohtaki, K. Eguchi, H. Arai, J. Mater. Chem. 7 (1), 85 (1997).

3. G. Heiland and E. Mollwo, Solid State Phys. 8, 191 (1959).

4. A. Weidenkaff, Adv. Eng. Mater. 6, 9, 709 (2004).

5. L. Bocher, M.H. Aguirre, D. Logvinovich, A. Shkabko, R. Robert, M. Trottmann, A. Weidenkaff, Inorg. Chem. 47, 8077 (2008).

6. R. Robert, S. Romer, A. Reller, A. Weidenkaff, Adv. Eng. Mat. 7, 303 (2005).

7. N. Schäuble, R. Dujardin, A. Weidenkaff, M. H. Aguirre, J. Electron. Mater. "Influence of the Thermal Aging Phenomena on the Thermoelectric Properties of Al-Substituted ZnO", submitted. 